\section{A. H. GLENN AND ASSOCIATES}

ALFRED H. GLENN

Certified Consulting Meteorologist

Consultants in Meteorology and Oceanography • Registered Engineers

in Louisiana and Texas

New Orleans Lakefront Airport

New Orleans, LA 70126

504-241-2222

TWX 810-951-6003

Telex 58-7445

Cable: GLENN, New Orleans

\section{WEATHER SERVICES CORPORATION}

JOHN E. WALLACE PETER R. LEAVITT JOHN P. MURPHY

\section{Certified Consulting Meteorologists}

Worldwide Forecasts, Climatological Surveys \& Data Banks for: Business

$\bullet$ Industry • Agribusiness • Marine • Media

131A Great Rd., Bedford, MA 01730

Bedford: 617-275-8860

New York: 212-267-1500

Houston: 713-738-1495

Telex $92-3330$

\section{NORTH AMERICAN WEATHER}

\section{CONSULTANTS}

KEITH J. BROWN, PRESIDENT

ROBERT D. ELLIOTT, CHIEF SCIENTIST

\section{Certified Consulting Meteorologists}

Weather Modification • Air Quality Surveys \& Field Studies • Applied Research $\bullet$ Forensic Meteorology

3761 South 700 East, Suite B

Salt Lake City, UT 84106

Santa Barbara, CA: 805-569-3754

801-263-3500

AEROMET, INC.

D. RAY BOOKER, PH.D., PRESIDENT

\title{
Certified Consulting Meteorologist
}

Instrumented Aircraft $\bullet$ Aerospace Meteorology

$R \& D \bullet$ Forecasts

P.O. Box 701767

Tulsa, OK 74170-1767

LOREN W. CROW CONSULTANTS, INC.

LOREN W. CROW, PRESIDENT

\section{Certified Consulting Meteorologist}

Consulting \& Applied Meteorological Services

3064 S. Monroe St.

Denver, CO 80210

303-753-6500 or

303-756-3971

MURRAY AND TRETTEL, INCORPORATED

JOHN R. MURRAY

JOHN P. BRADLEY

DENNIS W. TRETTEL

\section{Certified Consulting Meteorologists}

Founded 1946, Micro/Operational Forecasting $\bullet$ Media $\bullet$ Air Quality/ Meteorological/PSD Monitoring/Studies • Forensic Research • Dispersion Modeling • Nuclear Emergency Support

$414 \mathrm{~W}$. Frontage Rd.

Northfield, IL 60093

$312-446-7800$

\section{WEATHER CORPORATION OF AMERICA}

ARMAND R. IACCHEO

EUGENE G. GRUEBER

\section{Certified Consulting Meteorologists}

Operational Forecasts • Applied Research

5 American Industrial $\mathrm{Dr}$.

314-878-5150

St. Louis, MO 63043

Cable: Weather, St. Louis

\section{CLIMATOLOGICAL CONSULTING CORPORATION}

WALTER A. BOHAN

\section{Certified Consulting Meteorologist}

Specializing in Forensic Meteorology

P.O. Box 736

Park Ridge, IL $60068 \quad$ Asheville, NC 28805

312-825-3677

704-298-4237

\section{METRO MONITORING SERVICES}

ERWIN K. KAUPER

\section{Certified Consulting Meteorologist}

Environmental Impact \& Site Surveys $\bullet$ Air Pollution Field Studies $\bullet$ Diffusion Analysis $\bullet$ Weather \& Air Quality Forecasting $\bullet$ Air Pollution Monitoring Instrumentation

143 E. Rowland Ave.

Suite 6

Covina, CA 91723

818-332-8411

SIDNEY R. FRANK . . . GROUP

SIDNEY R. FRANK, PRESIDENT

\section{Certified Consulting Meteorologist}

Applied Environmental Research $\bullet$ Site Selection $\bullet$ Pollution Analyses • Wind/Solar Energy Assessments • Forensic Meteorology-Environment/ Weather Reconstruction at Air/Sea/Land Accident Sites

444 David Love $\mathrm{PI}$

Santa Barbara Municipal Airport

Goleta, CA 93117

805-964-4477

*For professional card rates, apply to: Executive Director, American Meteorological Society, 45 Beacon St., Boston, MA 02108. 


\section{RICHARD E. CALE}

\section{Certified Consulting Meteorologist}

Environmental Research Applications $\bullet$ Aviation Weather Investigations - Planning Services

P.O. Box 3070

Cerritos, CA 90703-3070

213-926-6149

\section{W. BOYNTON BECKWITH}

\section{Certified Consulting Meteorologist}

Aviation-Accident Investigation \& Operational Applications $\bullet$ Mesometeorological Analyses • Climatological Studies • Expert Testimony

14728 Caminito Orense Oeste

San Diego, CA 92129

$619-672-1565$

TRC ENVIRONMENTAL CONSULTANTS, INC.

GALE F. HOFFNAGLE

DOUGLAS R. MURRAY

DANIEL J. MCNAUGHTON

\section{Certified Consulting Meteorologists}

Environmental Consulting \& Research $\bullet$ Applied Meteorology $\bullet$ Air Quality $\&$ Meteorological Monitoring $\bullet$ Diffusion Modeling $\bullet$ Tracer Studies $\bullet$ Air Toxics Monitoring

800 Connecticut Blvd.

E. Hartford, CT 06108

203-289-8631

Denver, CO 303-792-5555

Somerset, NJ 201-563-1100

Los Angeles, CA 714-581-6860

Seattle, WA 206-485-2992

ERT (Environmental Research \& Technology, Inc.) DR. BRUCE A. EGAN

PAUL SHERR

ROBERT J. PAINE

\section{Certified Consulting Meteorologists}

Air Toxics Consulting $\bullet$ Clean Air Act Permitting, Compliance, and Variance Support - State-of-the-Art Modeling, Measurements, and Field Techniques • Hazardous Waste Services • Emergency Planning and Response • Expert Testimony

696 Virginia Rd., Concord, MA 01742

800-722-2440

Boston, MA; Pittsburgh, PA, Chicago, IL; Denver, CO; Dallas, TX; Houston, TX; Los Angeles, CA; Seattle, WA

APPLIED METEOROLOGY, INC.

JOHN W. HATHORN

\section{Certified Consulting Meteorologist}

Meteorological Consulting \& Analysis $\bullet$ Air Quality Modeling \& Monitoring

- Site Selection \& Permitting • Environmental Data Acquisition Systems

\& Network with Remote-Control

9000 Southwest Freeway

Suite 326

Houston, TX 77074

713-777-0106

P.O. Box 41008 Santa Barbara, CA 93140 805-965-2656 303-388-AERO

\section{WEATHER RESEARCH CENTER}

JOHN C. FREEMAN, DIRECTOR OF RESEARCH

LEON F. GRAVES, RESEARCH ASSOCIATE

\section{Certified Consulting Meteorologists}

Worldwide Weather \& Oceanographic Forecasting $\bullet$ Climatology $\bullet$ Expert Testimony $\bullet$ Research in Meteorology \& Oceanography $\bullet$ Wave Spectra - Software Development $\bullet$ Observing Network Design

3710 Mt. Vernon

Houston, TX 77006

713-529-3076

AEROVIRONMENT INC.

PAUL B. MACCREADY, JR., PH.D.

MICHAEL W. CHAN

\section{Certified Consulting Meteorologists}

Air Quality/Meteorology Hazardous Waste Monitoring $\bullet$ Diffusion Modeling • Tracer Experiments • Instrumentation-Acoustic Radar, Air Samplers $\bullet$ Wind Energy Assessments/Wind Engineering

825 Myrtle Ave.

Monrovia, CA 91016-3424 Santa Barbara, CA 805-967-7699

818-357-9983

Telex 467-121, Aerovir-Cl

St. Louis, MO 314-993-0543 Denver, CO 303-388-AERO

HALES AND CO.

J. VERN HALES, PH.D.

\section{Certified Consulting Meteorologist}

Environmental Consultants-Air Quality, Environmental Impact Studies, Field Programs, Remote Sensing, Atmospheric Physics

609 Lorient Dr.

West Chester, PA 19382-4970

215-696-4833

McVEHIL-MONNETT ASSOCIATES, INC.

GEORGE E. McVEHIL, PH.D

\section{Certified Consulting Meteorologist}

Industrial Meteorology $\bullet$ Air Quality Modeling $\bullet$ Permitting $\bullet$ Meteorological Analysis and Consulting Services

$5655 \mathrm{~S}$. Yosemite St.

Suite 104

Englewood, CO 80111

303-220-7213

\section{SIMPSON WEATHER ASSOCIATES, INC.}

R. H. SIMPSON, PH.D. G. D. EMMITT, PH.D. M. GARSTANG, PH.D.

\section{Certified Consulting Meteorologists}

Environmental Hazard/Risk Assessment $\bullet$ Hurricane Strike Probabilities - Weather Modification Research $\bullet$ Alternate Energy Studies $\bullet$ Field Measurements $\bullet$ Instrumentation Development

809 E. Jefferson St.

Charlottesville, VA 22902

804-979-3571

Certified Consulting Meteorologist: The certification program of the American Meteorological Society is aimed at fostering the establishment and maintenance of a high level of professional competency, and mature and ethical counsel, in the fields of consulting meteorology. Require- 


\section{INTERNATIONAL CENTER FOR THE SOLUTION OF ENVIRONMENTAL PROBLEMS \\ JOSEPH L. GOLDMAN, PH.D., TECHNICAL DIRECTOR \\ Certified Consulting Meteorologist}

Broad Scope Assessments by Interdisciplinary Experts Worldwide Specialize in Mesometeorological Variations • Climate Change $\bullet$ Problems Posed, Solutions Implemented \& Demonstrated

3818 Graustark

713-527-8711

Houston, TX 77006 Telex 775-167

\section{CLIMATOLOGICAL CONSULTING CORPORATION}

RALPH E. HUSCHKE

\section{Certified Consulting Meteorologist}

Specializing in Forensic Meteorology

P.O. Box 456

Coronado, CA 92118

Rt. 2, Box 372

(619) 435-5971 Asheville, NC 28805

(704) 298-4237

\section{DAMES \& MOORE}

PERRY W. FISHER

A. ROGER GREENWAY

\section{Certified Consulting Meteorologists}

Industrial Meteorology • Air Quality Modeling/Monitoring $\bullet$ PSD Permit Application Preparation - Expert Testimony $\bullet$ Climatological Studies • Nuclear Meteorological Services and Analyses

Park Ridge, IL

Cranford, NJ 312-297-6120

201-272-8300

AEROCOMP, INC.

JOSEPH A. CATALANO

\section{Certified Consulting Meteorologist}

Air Quality Monitoring \& Analysis $\bullet$ Atmospheric Modeling • Climatological Studies • Data Management Systems \& Software

3303 Harbor Blvd.

Costa Mesa, CA 92626

714-957-6596

\section{COLORADO INTERNATIONAL CORP.}

RALPH PAPANIA, JR.

\section{Certified Consulting Meteorologist}

Weather Modification Services $\bullet$ Cloud Physics Research $\bullet$ Radar Meteorology • Cloud Modeling • Airborne Instrumentation • Meteorology \& Air Quality Monitoring Stations

P.O. Box 3007

Boulder, CO 80307

303-443-0384 TWX 910-940-3444

\section{CLIMATOLOGICAL CONSULTING CORPORATION}

WILLIAM H. HAGGARD HENRY T. HARRISON

DR. WERNER A. BAUM

\section{Certified Consulting Meteorologists}

Specializing in Forensic Meteorology

Route 2, Box 372

Asheville, NC 28805

704-298-4237

OCEANROUTES, INC._a Swire Group Company JERRY A. WILLIAMS

\section{Certified Consulting Meteorologist}

Consultants in Meteorology \& Oceanography • Optimum Ship Routing • Site Specific Spectral Wave Forecasting

680 W. Maude Ave.

Sunnyvale, CA $94086-3518$

408-245-3600

TWX 910-373-1175

Telex 345-540

Cable OCEANROUTES

Aberdeen, Halifax, Hong Kong, Houston, London, N.Y., Perth, San Francisco, Singapore, \& Tokyo

\section{DAMES \& MOORE}

STANLEY J. KRIVO

GEORGE C. HOWROYD, PH.D.

JAMES W. LITTLE ROBERT G. ALLEN

\section{Certified Consulting Meteorologists}

Industrial Meteorology $\bullet$ Air Quality Modeling/Monitoring $\bullet$ PSD Permit Application Preparation • Expert Testimony • Climatological Studies • Nuclear Meteorological Services and Analyses

Atlanta, GA 404-262-2915

\section{$R^{\star}$ SCAN CORPORATION}

WALTER A. LYONS, PH.D., PRESIDENT

\section{Certified Consulting Meteorologist}

LPATS Lightning Data and Information Services - NVG GOES Image Animation Systems $\bullet$ Coastal/Mesoscale Numerical Models • Nuclear Emergency Response • Forensic Investigations

Business and Technology Center

511 Eleventh Avenue South

612-333-1424 Minneapolis, MN 55415 Telex 4310073 MEVBTC MPS

\section{WEATHER CONSULTANTS INCORPORATED}

\section{JOHN T. WALSER, PRESIDENT}

\section{Certified Consulting Meteorologists}

Specializing in Weather Modification Design \& Operations • Weather \& Climate Information Service and Expert Testimony $\bullet$ Data Acquisition \& Analysis • Wind Energy Assessment \& Siting

P.O. Box 3414

Santa Rosa, CA 95402

707-538-1080 Telex 38-0795

ments of knowledge, experience, and character are determined by a five-man Board. Objectives of the program and application procedures are described in full detail in the August 1987 Bulletin (pp. 975-978). 


\section{JERRY PARDUE}

\section{Certified Consulting Meteorologist}

Weather Investigations for Law Firms $\bullet$ Sunrise, Sunset \& Twilight $\bullet$ Expert Testimony

4065 Gilman Ave.

502-893-5551

Louisville, KY 40207

\section{LYLE E. BROSCHÉ}

\section{Certified Consulting Meteorologist}

Specializing in Long-Range Weather Outlooks and Consulting.

1347 Silver Lake Drive

Melbourne, FL 32940

305-259-1615

\section{NUS CORPORATION}

\author{
AMIRAM ROFFMAN, PH.D.
}

\section{Certified Consulting Meteorologist}

Air Quality Consulting, Diffusion Modeling, Air Toxics $\bullet$ Monitoring \& Field Programs • Environmental Studies, Hazardous \& Solid Waste Programs - Risk Assessment $\bullet$ Health \& Safety

Park West Two, Cliff Mine Road,

Pittsburgh, PA 15275-1071

Houston, TX; Gaithersburg, MD; Oak Ridge, TN; Holt, MI

J M SORGE, INC.

JOSEPH M. SORGE, PRESIDENT

\section{Certified Consulting Meteorologist}

Meteorological and Air Quality Modeling and Monitoring $\bullet$ Applied Climatology • Expert Testimony $\bullet$ Toxic and Hazardous Materials

3301 US Highway 22

Somerville, NJ 08876

201-218-0066

FREESE-NOTIS WEATHER, INC.

HARVEY FREESE, M.S.

\section{Certified Consulting Meteorologist}

Applied Forecasting for: Municipalities, State Government, Utilities, Radio \& Television, Commodities, Construction • Climatological Research, Expert Testimony, \& Forensic Meteorology

1453 NE 66th Ave.

Des Moines, IA 50313

$515-289-1314$

\section{AEROMATRIX INC.}

CONRAD J. MASON, PH.D., PRESIDENT

\section{Certified Consulting Meteorologist}

Solar Energy Surveys • Biometeorology - Meteorological \& Air Quality Monitoring • Dispersion Modeling • Site surveys $\bullet$ Expert Testimony

3640 E. Huron River Dr.

Ann Arbor, MI 48104

313-971-2244

\section{ATMOSPHERICS INCORPORATED}

THOMAS J. HENDERSON, PRESIDENT

\section{Certified Consulting Meteorologist}

Weather Modification Operation - Cloud Physics Research • Airborne Instrumentation $\bullet$ Weather Radar Applications $\bullet$ Aerosol Measurements - General Meteorological Services

5652 E. Dayton Ave.

Fresno, CA 93727

209-291-5575

ATMOSPHERIC RESEARCH \& TECHNOLOGY

EDWIN X. BERRY, PH.D., PRESIDENT

\section{Certified Consulting Meteorologist}

Atmospheric physics research. Wind energy evaluations. Atmospheric data systems. Data processing \& analysis

6040 Verner Ave.

Sacramento, CA 95841

$916-338-0550$

WILKENS WEATHER TECHNOLOGIES

\section{DIVISION}

Air Routing International Corporation

RICHARD B. WILKENS, EXECUTIVE VICE-PRESIDENT

\section{Certified Consulting Meteorologist}

Specialists in Marine, Aviation, \& Industrial Forecasting Worldwide $\bullet$ Hindcast \& Climatological Studies $\bullet$ Computerized Flight Plans

2925 Briarpark, Suite 610

713-977-0800

Houston, TX 77042

(Toll-Free) 800-231-5787

\section{COURTNEY CONSULTANTS, INC.}

F. E. COURTNEY, PRESIDENT

GEORGE W. REYNOLDS, PH.D.

\section{Certified Consulting Meteorologist}

Meteorology, Modeling, Monitoring, Permits, Noise, Dust, Storms, Air Quality, Testimony, Accidents, Severe Weather

520 Carriage Dr.

Atlanta, GA 30328

404-256-2487

Certified Consulting Meteorologist: The certification program of the American Meteorological Society is aimed at fostering the establishment and maintenance of a high level of professional competency, and mature and ethical counsel, in the fields of consulting meteorology. Require- 


\section{THE C. T. MAIN CORPORATION}

DAVID B. SMITH

HENRY A. CHARY

LAURENCE A. LABRIE

\section{Certified Consulting Meteorologists}

Air Quality/Meteorological Monitoring $\bullet$ Diffusion Modeling $\bullet$ Pollution • Analyses • Environmental Impact \& Site Surveys • Economic Feasibility - Hydro \& Engineering Meteorology

Prudential Center

Boston, MA 02199

Charlotte, NC

$617-262-3200$

GLOBAL WEATHER DYNAMICS, INC.

ARTHUR N. HULL, PH.D.

\section{Certified Consulting Meteorologist}

Worldwide Weather Services $\bullet$ Oceanographic Services $\bullet$ Forecasting $\bullet$ Modeling • Data Banks • Weather Message • Switching • Facilities Management $\bullet$ Applied Research $\bullet$ Turnkey Installations

2400 Garden Rd.

408-649-4500

Monterey, CA 93940 TWX 910-360-7081

GERALD W. GRAMS, PH.D.

\section{Certified Consulting Meteorologist}

Atmospheric Visibility, Aerosol Measurements, Laser Instrumentation, Computer Software Development, \& Consulting Services

\section{Cosmos Dr}

Atlanta, GA 30345

404-894-3897

\section{DAMES \& MOORE}

THOMAS A. UMENHOFER

\section{Certified Consulting Meteorologists}

Industrial Meteorology • Air Quality Modeling/Monitoring • PSD Permit Application Preparation • Expert Testimony $\bullet$ Climatological Studies • Nuclear Meteorology Services and Analyses

Santa Barbara, CA 805-685-4415

\section{METEOROLOGICAL STANDARDS INSTITUTE \\ THOMAS J. LOCKHART}

\section{Certified Consulting Meteorologist}

Quality Assurance Plans and Audits • System Specifications • Quality Assurance Training • Instrument Performance Testing • Certified Quality Engineer (A.S.Q.C.)

841 Seventh Lane

Fox Island, WA 98333

206-549-2179

\section{AIR SCIENCE CONSULTANTS, INC.}

RICHARD J. MANCINI, PRESIDENT

\section{Certified Consulting Meteorologist}

Forecasting Services for Industry, Government, \& News Media • Air Quality Modeling \& Monitoring • Stack Emissions Tests • Impact \& Climatological Studies • Forensic Meteorology

347 Prestley Rd.

Bridgeville, PA 15017

$412-221-6002$

\section{ATEK CORPORATION}

GEOFFREY E. HILL, PH.D., PRESIDENT

\section{Certified Consulting Meteorologist}

Atmospheric Technology \& Engineering $\bullet$ Weather Modification Research \& Operations $\bullet$ Remote Sensing $\bullet$ Computer Applications

900 28th Street, Suite 202

Boulder, CO 80303

$303-449-5588$

\section{WEATHER APPLICATIONS}

DUANE R. (BOB) GOODMAN

\section{Certified Consulting Meteorologist}

Meteorology for Hazardous Waste Site Remediation, Climatology, Air Quality, Consulting, Forensic Meteorology

8520 41st West

Tacoma, WA 98466

$206-564-3724$

\section{WEATHERDATA, INC.}

MIKE SMITH, PRESIDENT

DANIEL R. BOWMAN, DIRECTOR OF OPERATIONS

\section{Certified Consulting Meteorologists}

Meteorological Investigations Relating to Legal Matters • Expert Testimony $\bullet$ Specialized Forecasting $\bullet$ Broadcast Meteorology and Newspaper Meteorology $\bullet$ Weather Photography and Graphics

833 North Main St.

Wichita, KS 67203

$316-265-9127$

GALSON TECHNICAL SERVICES, INC.

R. LELAND DAVIS, VICE PRESIDENT

ALAN J. KROL

\section{Certified Consulting Meteorologists}

Consulting and Services in the Environmental Sciences

Dispersion Modeling $\bullet$ AIR-1(C) Air Toxics Management \& Assessment Program • AQ/Meteorological Monitoring • Source Testing • Industrial Hygiene $\bullet$ Expert Testimony $\bullet$ Permitting $\bullet$ Complete Laboratory Services 6601 Kirkville Road Rochester, NY 716-235-2220 E. Syracuse, NY $13057 \quad$ Plymouth Mtg., PA 215-834-7288 315-432-0506 Oakland, CA. 415-763-1677

ments of knowledge, experience, and character are determined by a five-man Board. Objectives of the program and application procedures are described in full detail in the August 1987 BullETIN (pp. 975-978). 


\section{METEOROLOGICAL APPLICATIONS}

JAMES H. MEYER, PRESIDENT

\section{Certified Consulting Meteorologist}

Forensic and Applied Meteorology - Climatology \& Environmental Research • Meteorological \& Air Quality Instrumentation \& Monitoring • Fugitive Dust \& Cooling Tower Drift Studies • Expert Testimony

12926 Allerton Lane

Silver Spring, MD 20904

301-384-1875

\section{ALEXIS B. LONG, PH.D. \\ Certified Consulting Meteorologist}

Weather Modification and Cloud Physics Research • Hail Measurement

193 Tramway Parade

Beaumaris, Victoria 3193

Australia

(61)3-589-5103

\section{METEOROLOGICAL EVALUATION}

SERVICES, CO. INC. (MES)

PATRICK T. BRENNAN, PRESIDENT

JOHN R. MARTIN, VICE PRESIDENT

\section{Certified Consulting Meteorologists}

Air-Quality Consulting $\bullet$ Expert Testimony $\bullet$ Industrial Meteorology $\bullet \mathrm{Nu}$ clear Licensing Studies • Weather Investigations For Legal and Insurance Firms

165 Broadway

Amityville, NY 11701

516-691-3395

\section{CLIMATOLOGICAL CONSULTING CORPORATION}

DR. VANCE A. MYERS

\section{Certified Consulting Meteorologist}

Specializing in Forensic Meteorology

9900 Mosby Road

Fairfax, VA 22032

(703) 273-1977

Rt. 2, Box 372 Asheville, NC 28805

(704) 298-4237

ROBERT L. PEACE, JR.

\section{Certified Consulting Meteorologist}

Industrial Meteorology $\bullet$ Micro-Mesoscale Modeling and Climatological Studies • Remote Sensing Studies • Software Development

2050 New York Drive

Altadena, CA 91001

818-798-3435

\section{EINAR L. HOVIND \\ Certified Consulting Meteorologist}

Industrial Meteorology $\bullet$ Air Quality Analysis $\bullet$ Weather Modification Services $\bullet$ Climatology $\bullet$ Forensic Meteorology

4152 Primavera Rd.

Santa Barbara, CA 93110

805-964-8218

WEATHER NETWORK, INC.

dba NOWCASTING

an Oceanroutes Company

DONALD F. SCHUKRAFT

\section{Certified Consulting Meteorologist}

Operational Weather Forecasts \& Climatological Studies $\bullet$ Instrumentation - Forensic Meteorology $\bullet$ Direct-Access Real-Time Meteorological Data Base

3760 Morrow Lane, Suite $F$

Chico, CA 95928-8865

916-893-0308

\section{SIGMA RESEARCH CORPORATION}

DR. STEVEN R. HANNA

DR. LLOYD L. SCHULMAN

\section{Certified Consulting Meteorologists}

Research and Consulting in the Environmental and Physical Sciences • Transport and Dispersion Modeling • Data Analysis • Tracer Studies • Permitting • Expert Testimony

394 Lowell St., Suite 12

Lexington, MA 02173

$617-862-0920$

\section{RAY HOXIT, PH.D. \\ Certified Consulting Meteorologist}

Detailed Weather Analyses $\bullet$ Forensic Meteorology and Expert Testimony - Climatological Studies • Specialized Weather Forecasts

P.O. Box 836

Arden, NC 28704

$704-891-4902$

\section{SPENCER DUCKWORTH}

\section{Certified Consulting Meteorologist}

Weather-Related Marine Incident Evaluations $\bullet$ USCG Licensed, Vessel Operator $\bullet$ Registered Engineer in California

2506 Seville Ct.

Davis, CA 95616

$916-756-9558$

Certified Consulting Meteorologist: The certification program of the American Meteorological Society is aimed at fostering the establishment and maintenance of a high level of professional competency, and mature and ethical counsel, in the fields of consulting meteorology. Require- 
ROY F. WESTON, INC.

PAUL M. FRANSIOLI

\section{Certified Consulting Meteorologists}

Ambient Air Quality and Meteorological Monitoring and Modeling • Industrial and Utility Air Permitting - Air Toxics Risk Assessment $\bullet$ Industrial Meteorology • Expert Testimony

Weston Way

West Chester, PA 19380

100 Corporate North, Suite 101

215-692-3030

Route 22 and Lakeside Drive

Bannockburn, IL 60015 $312-295-6020$
STONE \& WEBSTER ENGINEERING CORP.

T. J. BURDA

C. A. MAZZOLA

A. KASPRAK

S. A. VIGEANT

\section{Certified Consulting Meteorologists}

Meteorological System Audits - Hazardous Chemical Modeling Impact Evaluations • New Source Review Permitting • Climatological Studies • Risk Assessment $\bullet$ Expert Testimony

P.O. Box 2325

Boston, MA 02107

5555 Greenwood Plaza Blvd.

Englewood, CO 80111-2113

617-589-2701 303-741-7440

Other offices in New York, NY; Cherry Hill, NJ; Houston, TX; Ft. Lauderdale, FL; Tampa, FL.

ments of knowledge, experience, and character are determined by a five-man Board. Objectives of the program and application procedures are described in full detail in the August 1987 Bulletin (pp. 975-978). 


\section{professional directory}

MCFARLAND WEATHER SERVICE Founded 1956

CHARLES J. FORD, PRESIDENT

Media, Industrial \& Aviation Forecasts $\bullet$ Long-Range Seasonal Outlooks - Air Pollution Studies • Investigations for Law Firms \& Insurance Companies • Expert Testimony

Allegheny County Airport

W. Mifflin, PA 15122

$412-462-6464$

\section{INTERNATIONAL WEATHER CORPORATION}

WALTER F. ZELTMANN

Forensic Meteorology • Applied Climatology

719 Bay Ridge Ave.

Brooklyn, NY 11220

718-748-8066

\section{BENDIX FIELD ENGINEERING CORP.}

ROBERT A. RAGUSO, MANAGER

\section{Marine Science Services}

Optimum Ship Routing $\bullet$ Ocean Weather Forecasts $\bullet$ Marine Operations Consulting

P.O. Box 2205

S. Hackensack, NJ 07606

212-947-9000

201-288-1550

TWX: 710-990-6114

Telex: 134-318

Cable: BEXMARSERV

\section{CONTROL DATA CORPORATION}

Meteorological Research and Consulting

\section{Consulting Services}

Atmospheric Dynamics Research $\bullet$ Middle Atmosphere Circulation $\bullet$ Trace Constituents $\bullet$ Remote Sensing $\bullet$ Data Management and Display $\bullet A r-$ tificial Intelligence/Expert Systems

P.O. Box 1249

Minneapolis, MN 55440 $612-853-3598$

ACCU-WEATHER, INC.

JOEL N. MYERS, PH.D., PRESIDENT

ELLIOT ABRAMS, M.S., CCM, SENIOR VICE-PRESIDENT

JOSEPH P. SOBEL, PH.D., SENIOR VICE-PRESIDENT

Weather Forecasting $\bullet$ Real Time Meteorological Data Base $\bullet$ Satellite, Radar, and Graphics Images • Climatological and Forensic Services • Over 20 years of quality service

619 W. College Ave.

State College, PA 16801

814-237-0309

\section{NATIONAL WEATHER CORPORATION Worldwide Weather Services}

Aviation Meteorology $\bullet$ Computer Flight Planning $\bullet$ Industrial Meteorology

- Data Acquisition \& Analysis • Climatological Research • Commodities

- Expert Testimony

Pan Am Bldg.

Teterboro Airport

Teterboro, NJ 07608

914-761-7600

\section{WEATHER SURVEYS}

CONRAD B. GOSSET, M.S., FORENSIC METEOROLOGIST

Specializing in Investigations for Law Firms \& Insurance Companies

Bank of New York Bldg

P.O. Box 473

Pleasantville, NY 10570

$914-769-1350$

\section{GULF COAST WEATHER SERVICE \\ WTVT Television Weather Service}

ROY LEEP, DIRECTOR

Complete Computerized Data Gathering $\bullet$ Video Graphics $\bullet$ Digital Nowcasting $\bullet$ Industrial Forecasting

P.O. Box 22013

Tampa, FL 33622

TWX: 810-876-0618

813-876-3592 Telex: 52679

\section{GEOMET TECHNOLOGIES, INC.}

\section{MARK J. STUNDER}

Environmental Expert Systems, Artificial Intelligence $\bullet$ Air Pollution Analysis, Modeling \& Monitoring • Environmental Data Systems, Climatological Studies • Research and Operations

20251 Century Boulevard

Germantown, MD 20874

$301-428-9898$

\section{CENTRAL WEATHER SERVICE}

EARL S. FINCKLE, PRESIDENT

\section{Consulting Meteorologists}

Radio Weathercasting Experts \& Commodity Weather Specialists $\bullet$ Aviation, Business \& Industry, Government, Forensic Meteorology • Over a Quarter of a Century of Experience

*For professional card rates, apply to: Executive Director, American Meteorological Society, 45 Beacon St., Boston, MA 02108. 


\section{KNIGHT-RIDDER/GLOBAL WEATHER SERVICES}

Crop Weather Impact Analysis via: Knight-Ridder Financial News $\bullet$ KnightRidder Financial Information - Commodity News Services • American Quotation Systems • UNICOM Inc.

P.O. Box 6053

Leawood, KS 66206

FLEETWEATHER, INC.

Since 1969

TORE H. JAKOBSEN, PRESIDENT, M.S., CO-OWNER

Forecasts for Weather-Sensitive Industry - Past Weather Research \& Expert Testimony $\bullet$ Global Ship-Guidance \& Surveillance $\bullet$ Weathercasting $\bullet$ Weather Data Management Software

The Fleetweather Building, Rte 52

RD9 Box 310

Hopewell Jct., NY $12533 \quad$ TWX 914-226-8200

914-226-8200 Telex 646679

\section{METEOTEC INC.-An Alaska Science \&} Technology Co.

ROBERT L. BALDWIN, PRESIDENT

\section{Environmental Science \& Engineering Consultants}

Analysis • Systems • Sensing $\bullet$ Communications $\bullet$ Automation $\bullet$ Wind \& Solar Energy • Air Quality $\bullet$ Noise $\bullet$ Modeling $\bullet$ Forensics $\bullet$ Management $\bullet$ Planning $\bullet R \& D$

P.O. Box 102915

907-562-2482

Anchorage, AK 99510 Telex 09026713 AKSCITECH AHG

\section{BOYD E. QUATE \& ASSOCIATES}

Specializing in Extended Period Weather Outlooks

P.O. Box 7065-Holland Station

Suffolk, VA 23437

COMPU-WEATHER, INC.

\section{Forecast \& Research Specialists}

Weather Forecasting for Media, Industry, Government $\bullet$ Research Studies - Consultation $\bullet$ Forensic Meteorology $\bullet$ Excellent Client References

29-50 Union St.

Flushing, NY 11354

\section{W. THORNTHWAITE ASSOCIATES}

WILLIAM J. SUPERIOR, PRESIDENT

Research \& Field Studies • Sensors \& Systems • Publications in Climatology

Rte. 1, Centerton

Elmer, NJ 08318

$609-358-2350$

\section{BRUCE F. WATSON}

\section{Consulting Meteorologist}

Research Specializing in Weather \& Climate of Minnesota \& Bordering States \& Provinces for Application to Agriculture, Forensics, Engineering Studies, Environment, \& Marketing

2514 Brenner St.

Roseville, MN 55113

$612-633-3496$

NASH C. ROBERTS, JR., CONSULTANTS, INC.

\section{Consulting Meteorologists Since 1946}

General Industrial $\bullet$ Marine $\bullet$ Forensic $\bullet$ Air Quality $\bullet$ Agricultural $\bullet$ Com modities • Television

1040 N. Rampart St.

TWX 810-951-5272

New Orleans, LA 70176 and $810-951-5273$

504-581-1688 Telex 58-4249

WEATHERBANK, INC.

MARK E. EUBANK, PRESIDENT

STEVEN A. ROOT, CCM BARRY C. NIELSEN

Operational Forecasting $\bullet$ Broadcast Services $\bullet$ Satellite Transmission Network • Forensic Studies • Real Time Meteorological Data Base • Climatological Research

2185 South 3600 West

Salt Lake City, UT 84119

801-973-3131

\section{WEATHER CONSULTANTS OF CANADA} CO. LTD. (TELEWEATHER)

MORRIS KESTIN, PRESIDENT

Long-Range Weather Outlooks \& Detailed Forecasts for Marketing, Radio, TV, Movies, Publications, Commodities. Consultants to Industry, Municipalities, Utilities, Forensic Meteorology since 1956

65 Marlborough Ave

416-968-7981

Toronto, Ont., Canada M5R 1X5 416-968-6664 


\section{YORK RESEARCH CORPORATION}

\section{Department of Atmospheric Sciences}

BRUCE R. WUEBBER

EDWARD J. KAPLIN

Air Pollution Meteorology $\bullet$ Atmospheric Diffusion Modeling $\bullet$ Meteorological/Air Quality Monitoring Systems • Boundary-Layer Profiling

1 Research Dr.

Stamford, CT 06906

203-325-1371

\section{MATRIX MANAGEMENT GROUP}

\section{General Management Consultants}

Meteorological Consultants to Government \& Industry $\bullet$ Project Management $\bullet$ Organization $\bullet$ Mgmt. audits $\bullet$ Mgmt. training $\bullet$ Policy Studies $\bullet$ Bridging the Gap Between Meteorology and Public Works

466 Colman Bldg. Seattle, WA 98104 206-621-1977 206-842-4186

\section{ASSOCIATED WEATHER SERVICES, INC.}

BRUCE H. BAILEY, VICE PRESIDENT

Applied Climatology $\bullet$ Atmospheric Field Research $\bullet$ Litigation $\bullet$ Wind Energy Meteorology • Specialized Forecasting • Environmental Impact Analyses

55 Colvin Ave.

Albany, NY 12206

$518-458-2263$

\section{ESSCO-ENVIRONMENTAL SCIENCE AND SERVICES CORPORATION}

\section{WALTER G. HOYDYSH, PH.D., DIRECTOR}

Wind Tunnel Modeling • GEP Stack Height Demonstration • Dispersion Modeling $\bullet$ Ambient Air Quality Monitoring $\bullet$ Pedestrian Level Wind Studies $\bullet$ Wind Pressure \& Aeroelastic Studies

45-43 37th St.

Long Island City, NY 11101

718-786-3948

\section{EARTH ENVIRONMENT SERVICE}

STEVE NEWMAN, CHIEF METEOROLOGIST

Agriculture $\bullet$ Site Forecasts $\bullet$ Media $\bullet$ Graphics $\bullet$ Expert Testimony $\bullet$ Worldwide Forecasting

\section{LIGHTNING LOCATION \& PROTECTION,} INC.

Lightning Locating \& Warning Systems • Lightning \& Weather Radar Display Systems • Sensors for Automatic Meteorological Stations • Consulting on Lightning Hazards \& Lightning Protection

1001 S. Euclid Ave.

Tucson, AZ 85719

$602-624-9967$

\section{WSI CORPORATION}

TODD GLICKMAN, MANAGER, GOVERNMENT PROGRAMS

ARLO W. GAMBELL, CCM

Real-Time Meteorological Data and Information - Digital Satellite and Meteorological Graphics • Service to Industry, Academia, Government, Media, Aviation, Energy, Agribusiness

41 North Road

617-275-5300

Bedford, MA 01730

Telex 95-1184

\section{NORTEC, A DIVISION OF ERT, INC.}

JOSEPH E. OSTROM, MANAGER OF METEOROLOGICAL SERVICES

Operational Marine Forecasting $\bullet$ Aviation Meteorology $\bullet$ Data Acquisition \& Analysis $\bullet$ Specializing in the Alaskan \& the Offshore Area

750 W. 2nd Ave.

Anchorage, AK 99501

$907-276-4302$

ENVIRONMENTAL SATELLITE DATA, INC.

JIM MENARD, METEOROLOGIST/SUPPORT MANAGER

ALLAN LANG, METEOROLOGIST

JIM KLEIN, METEOROLOGIST

Digital Satellite Images • Weather Graphics • Computer Graphics Systems for TV, Aviation, Industry, and Government with Interface to Radar, Doppler Radar, and Lightning Detection.

World Weather Building, 1st Floor

5200 Auth Road

Suitland, MD 20746

$301-423-2113$

FRED W. DECKER, PH.D.

Meteorologist Since 1937 (USWB, AAF, USAFR, Universities, Forensic Expert).

Defense Attorneys' Expert Consultant: Weather Reconstructions • CrossExamination Advice $\bullet$ Expert Testimony $\bullet$ Background: Forecaster $\bullet$ Professor • Author ("The Weather Workbook")

827 NW 31st St.,

Corvallis, OR 97330

503-753-7271 


\section{METEOROLOGICAL EVALUATION}

SERVICES, CO. INC. (MES)

MAYNARD E. SMITH

FRANK P. CASTELLI

\section{Consulting Meteorologists Since 1968}

Air-Quality Modeling and Analysis • Forensic Meteorology $\bullet$ Monitoring and Data Analysis • Nuclear and Industrial Permitting Studies

165 Broadway

Amityville, NY 11701

\section{METRO WEATHER SERVICE, INC.}

\section{CRAIG ALLEN, VICE PRESIDENT}

Servicing our clients for 15 years

Specialized Forecasts for the Media, Utilities \& Industry $\bullet$ Research \& Consultation • TV Weathercaster Training

Hangar \#11@ JFK Airport

Jamaica, N.Y. 11430

718-917-6565

\section{TECHNI-WEATHER CENTER}

\section{WILLIAM JACQUEMIN, CHIEF METEOROLOGIST}

Weather Forecasting: Media $\bullet$ Utilities $\bullet$ Industry $\bullet$ Government $\bullet$ Oceanographic Research • Education Services • Weatherphones • CoastalManagement Reports • Environmental-Impact Studies

P.O. Box \#2353

203-744-2064

Danbury, CT 06813-2353

203-792-9500

\section{ALLIED WEATHER CONSULTANTS}

Weather Forecasting Services For Utilities, Agriculture, Construction, and the Film Industry

143 E. Rowland Ave.

Suite 6

Covina, CA 91723

818-967-1098

BALL TROPICAL METEOROLOGICAL

\section{SERVICES}

\section{Weather Forecasting and Research}

ROGER S. BALL, JR.

\section{Consulting Meteorologist}

Forecasts for Utilities, Marine, Agriculture, Construction, Aviation $\bullet$ Specialists in Tropical and Satellite Meteorology • Forensic Meteorology/ Litigation-Expert Testimony • Climatology • Air-Pollution Meteorology

\section{WILLIAM C. FRANCE}

Meteorologist more than 35 years (military, airline, government)

Expert advice or testimony

1420 Centre Ave. \#1815

Pittsburgh, PA 15219

412-391-1083

\section{SURFACE SYSTEMS, INC.}

JOE R. KELLEY, VICE-PRESIDENT,

METEOROLOGY/TECHNOLOGY

DAVID C. TRASK, MANAGER, FORECASTING SERVICES

Real-Time Surface (Pavement) Condition Monitoring Systems $\bullet$ Detailed Roadway and Runway Temperature Projections and Weather Impact Forecasts $\bullet$ Real-Time Color Radar Systems $\bullet$ Computer Access Software for NWS Radars - Tailored Color Graphics for Monitoring Surface and Atmospheric Sensors $\bullet$ Computerized Weather Access Service

2605 S. Hanley Road

314-781-6501

St. Louis, MO 63144 Telex 503744

WEATHER TRADES, INC.

JAMES ROEMER, PRESIDENT

Specializing in Commodity Trading \& Hedging Strategies Based on World Weather Conditions $\bullet$ Managed Account Programs $\bullet$ Commodity Advisory Services • Trade Winds Newsletter

P.O. Box 244

Ankeny, IA 50021

$515-289-2470$

\section{IMET CORPORATION}

RICHARD I. CROUTHAMEL

DALE F. BRYAN

Meteorological Observation Systems • Environmental Studies/Wind \& Solar Surveys • Forensic Meteorology/Legal Testimony

703-777-0711 